Revista de Matemática: Teoría y Aplicaciones 2011 18(1) : 63-76

CIMPA - UCR ISSN: $1409-2433$

\title{
USO DE UN AUTÓMATA CELULAR PARA CREAR UN MODELO DE DIFUSIÓN DE CONTAMINANTES EN UN SISTEMA \\ SUELO-AGUA
}

\author{
CREATION OF A MODEL OF POLLUTANS \\ DIFFUSION IN SOIL-WATER SYSTEM USING A \\ CELLULAR AUTOMATA \\ Glicina Merino-Castro*
}

Received: 18 Feb 2010; Revised: 18 Nov 2010;

Accepted: 26 Nov 2010

*Escuela de Ingeniería y Arquitectura, Tecnológico de Monterrey, Campus Toluca, Toluca, México. E-Mail: merino@itesm.mx 


\title{
Resumen
}

En este trabajo se presenta el desarrollo de un modelo de difusión de contaminantes en un sistema suelo-agua bajo condiciones específicas de un medio real en estudio. El propósito de dicho modelo es permitir la observación del flujo del contaminante en el sistema suelo-agua y su comportamiento al interactuar con los diferentes elementos del sistema con la finalidad de orientar y apoyar los procesos y la aplicación de las técnicas de recuperación de suelos y aguas contaminadas. Las condiciones básicas para el diseño del modelo son: 1) El contaminante es introducido en el suelo. 2) El contaminante fluye del suelo al agua. 3) La concentración del contaminante introducido es variable durante el proceso. 4) El contaminante puede llegar a saturar el suelo. 5) El contaminante es adsorbido por el suelo. 6) El modelo se realiza en 2D, con posibilidad de extenderlo a 3D. El modelo utiliza un autómata celular, el cual contiene diferentes zonas con sus propias reglas de funcionamiento. Se consideran cuatro zonas en el modelo: i) Zona de introducción del contaminante. ii) Zona del suelo (un medio poroso). iii) Zona de interrelación del suelo-agua. iv) Zona del agua.

Palabras clave: autómata celular, difusión de contaminantes, sistema suelo-agua, medio poroso.

\begin{abstract}
In this paper is presented a developed model for a pollutant diffusion in a soil-water system, for specific conditions in a real study area. A model that allows the observations of the pollutant flow in the soil-water system and its behavior and interaction with the different system elements, is searched with the purpose of orientate and support the processes and techniques application for the pollutant soil and water remediation. The basic model conditions are: 1) The pollutant is introduced to the soil. 2) The pollutant flows from the soil to the water. 3) The introduced pollutant concentration is variable during the process. 4) The pollutant may saturate the soil. 5) The pollutant is adsorbed by the soil. 6) The model is developed in $2 \mathrm{D}$, with the possibility to be expanded to 3D. The model uses a cellular automata that contains different zones with their own function rules. There are four considerate zones: i) Introduction of the pollutant zone. ii) Soil zone (a porous media). iii) Soil-water interaction zone. iv) Water zone.
\end{abstract}

Keywords: cellular automata, pollutant diffusion, soil-water system, porous media.

Mathematics Subject Classification: 68U20.

Rev.Mate.Teor.Aplic. (ISSN 1409-2433) Vol. 18(1): 63-76, January 2011 


\section{Introducción}

Los flujos subterráneos que se presentan en los proyectos de remediación ambiental, se ven afectados por heterogeneidades de diferentes tipos y a diferentes escalas del transporte del fluido. El obtener una solución numérica a partir de una expresión matemática para dicho fenómeno es bastante difícil. Naturalmente existen diferentes formas de aproximarse al problema del modelado matemático del transporte de fluido; los métodos tienen sus propias y diferentes limitaciones. En algunos, las condiciones globales de frontera hacen perder robustez o disminuir la precisión del modelo, éste se vuelve tosco y perdemos el uso de procedimientos numéricos generales apropiados.

Aquí se presenta el uso de un Autómata Celular para modelar el flujo de contaminantes en un sistema suelo-agua. El trabajo esta dividido de la manera siguiente: en la sección 2 se describen las características y condiciones generales del sistema físico que se pretende modelar, la sección 3 plantea el porqué del uso de un Autómata Celular. En la sección 4 se analiza y describe la implementación del Autómata Celular, su funcionamiento se encuentra en la sección 5. Finalmente se tienen las conclusiones.

\section{Características y condicionantes generales del sistema físico a modelar}

El sistema físico que se busca modelar consiste en un suelo que recibe fluidos contaminantes y se encuentra próximo a un cuerpo de agua.

El modelo de difusión de contaminantes en el sistema suelo-agua debe cumplir las condiciones iniciales siguientes:

1. El contaminante es introducido en el suelo.

2. El contaminante fluye del suelo al agua.

3. La concentración del contaminante introducido es variable durante el proceso.

4. El contaminante puede llegar a saturar el suelo.

5. El contaminante es adsorbido por el suelo.

6. El modelo se realiza en 2D, con posibilidad de extenderlo a 3D.

Se busca que el modelo permita la observación del flujo del contaminante en el sistema y su comportamiento al interactuar con los diferentes 
elementos del sistema permitiendo a la vez, orientar los procesos de experimentación en el sistema físico real. Estas condicionantes nos indican que, además de obtener algunos resultados numéricos, será importante observar una evolución gráfica del modelo.

En el sistema físico observamos 4 grandes zonas perfectamente distinguibles, estas son:

1. Zona de introducción del contaminante.

2. Zona del suelo (un medio poroso).

3. Zona del agua.

4. Zona de interrelación de suelo y agua.

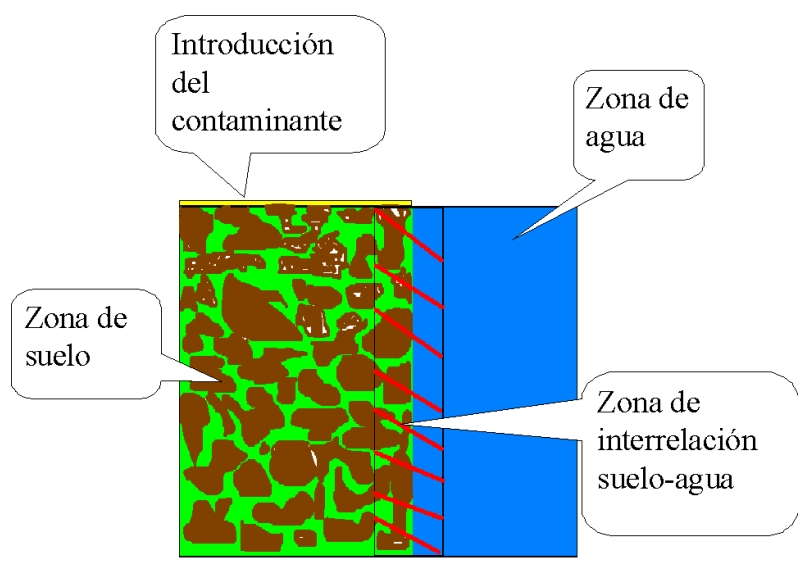

Figura 1: Esquema del sistema físico a modelar.

\section{3 ¿Por qué un autómata celular?}

Las aplicaciones de los Autómatas Celulares son muy variadas, desde simulaciones microscópicas de la Física y la Biología hasta simulaciones macroscópicas de procesos geológicos o sociales.

La evolución de los fenómenos complejos puede ser descrita en términos de las interacciones locales de sus componentes, un modelado eficiente de dichos fenómenos está basado en los Autómatas Celulares. Un Autómata Celular es un autómata finito consistente en un espacio dividido en celdas; cada una de ellas contiene las condiciones de su estado, el cual es modificado por el estado de las celdas en su vecindad. En el tiempo $t=0$ las 
celdas se encuentran en un estado arbitrario, que representa las condiciones iniciales del sistema. Dentro del Autómata Celular se tiene una función de transición de estado para cada zona con iguales características, esta función se aplica simultáneamente a cada una de las celdas. La evolución del Autómata Celular cambia los estados de todas las celdas simultáneamente en tiempos discretos, de acuerdo a las funciones de transición.

El sistema físico que se considera aquí es un campo de aplicación potencial para un Autómata Celular, ya que el sistema evoluciona exclusivamente a través de interacciones locales; por lo cual se propone la creación de un modelo basado en un Autómata Celular con reglas de evolución diferentes para cada zona representativa del sistema físico.

\section{El modelo de autómata celular para un sistema suelo-agua}

\subsection{Zona de introducción del contaminante}

Esta zona permite la entrada del flujo contaminante con la concentración deseada (condición 1), pudiendo cambiarse dicha concentración durante el desarrollo de la simulación o en diferentes simulaciones (condición 3). La zona se considera actuando únicamente sobre la tierra y no introduce fluido contaminante sobre la zona del agua (condición 1). La introducción del flujo en el medio poroso sigue la dirección de la gravedad.

\subsection{Zona del suelo. Un medio poroso}

Un medio poroso es una estructura (matriz) de granos y tubos estrechos (poros) por donde el fluido circula. El comportamiento de un fluido en este tipo de medio es diferente en cada uno de sus componentes.

El suelo se caracteriza como un medio poroso en el cual el flujo del contaminante se produce en los poros y la adsorción-desorción del contaminante en los granos (parte sólida del suelo).

La descripción del flujo en un medio poroso es bastante difícil dada la complejidad de la estructura de un medio de este tipo. Aunque se tiene una representación matemática para el flujo de un fluido en un solo tubo, en una red de tubos es imposible determinar a detalle la circulación de un fluido.

En los poros el flujo del contaminante tiene un comportamiento de difusión y advección mientras el suelo no esté saturado. En el momento de la saturación no se presenta ningún movimiento en los poros, salvo que tengamos diferencia de concentraciones en el flujo lo cual provoca movimientos 
de convección (puede provocarse con el cambio de la concentración del contaminante a lo largo de una misma simulación).

La condición 5 (El contaminante es adsorbido por el suelo) implica la adsorción y permanencia del contaminante en los granos, así como su desorción. En la bibliografía correspondiente, se tienen cuantificaciones de los coeficientes de adsorción de distintos tipos de suelo y la vida media de diferentes contaminantes.

\subsubsection{Generación del medio poroso}

La porosidad en un medio poroso se define como:

$$
\phi=\frac{\text { volumen de poros }}{\text { volumen de la matriz }}
$$

en donde el volumen de poros es el volumen total del conjunto de poros en la matriz y el volumen de la matriz es el volumen total de la estructura del medio poroso. Entonces tenemos que $0 \leq \phi \leq 1$. Normalmente se considera que la porosidad es constante en todo el medio poroso.

Para la simulación de la porosidad se sigue un procedimiento que genera en 2D una estructura con conectividad interna compleja. La idea es distribuir los espacios o los obstáculos de manera aleatoria en la región, usando un número apropiado de la función de distribución de la densidad $[2]$.

Los granos y los poros de rocas y sólidos frecuentemente siguen una distribución log-normal. Aunque se ha observado que muchos tienen una distribución fractal en sus poros a pequeña escala (menor que 1/100 de micra) [18]. En un medio fractal, el número de densidad m para un elemento de tamaño $L$ está dado por:

$$
m(L)=A L^{2-D}
$$

donde $D$ es la dimensión fractal y $A$ es un coeficiente que depende del material sólido.

El modelo se restringe a medios con características fractales, aunque puede describir una distribución log-normal para elementos grandes y una distribución fractal para escalas de pequeñas a intermedias.

El algoritmo seguido es:

(a) Construir un conjunto de obstáculos (granos). Los tamaños deben estar en el rango de validez de (1). Típicamente, cada tamaño sucesivo se toma dividiendo el tamaño previo entre 2. 
(b) Determinar la densidad para cada obstáculo del tamaño usado en (1). Calcular:

$$
\Delta=\Delta x=\Delta y=\Delta z=\frac{1}{m(L)} .
$$

Dividir la región de interés en subdominios de tamaño $N \Delta x, N \Delta y \mathrm{y}$ $N \Delta z$ donde $N$ es proporcionado por el usuario. El resto de la región debe ser un múltiplo entero de $N \Delta$. El número esperado, $E(L)$, de cada obstáculo de tamaño $L$ en cada subdominio es $N^{3}$.

(c) En cada uno de los subdominios, el número esperado de elementos de tamaño $L$ se distribuyen aleatoriamente. La forma de los elementos puede ser rectangulares o circulares (en 2D), cúbica o esférica (en 3D). En nuestro caso se trabajará en 2D y los elementos serán rectangulares. Las formas de mayor tamaño se distribuyen primero. Las formas pequeñas se pueden traslapar con las mayores ya distribuidas. Se restringe la localización de los elementos a los subdominios. La estructura obtenida debe ser característica del material que se va a describir. Los valores $\Delta x, \Delta y, \Delta z$ usualmente son más grandes que el correspondiente $L$. (Nota: la restricción de colocar los elementos en un subdominio no es una restricción dominante).

(d) La naturaleza de cada elemento sea un grano o un poro, se determina probabilísticamente. Inicialmente todos son granos.

Para el control de la porosidad final se utiliza una constante $b$, entre 0 y 1 , que es el valor de la porosidad deseada en la simulación. Un elemento es declarado grano si:

$$
\min (1, \operatorname{int}(\operatorname{rand} / b))=1
$$

de otro modo representa un poro. En (3) rand es un número aleatorio entre 0 y 1 , int es la función mayor entero.

El procedimiento anterior produce una estructura con una porosidad cercana a $b$.

\subsubsection{Generación de la adsorción-desorción en los granos}

En esta sección se ha tomado en cuenta que si el sustrato adsorbente está inicialmente vacío, después de un tiempo el sustrato se saturará o alcanzará una adsorción plena; además si el sustrato presenta una desorción eficiente, se previene la saturación de dicho sustrato. Por otra parte, es importante obtener resultados numéricos y gráficos del comportamiento del fluido en su trayecto por la zona del suelo y en particular de los granos. 


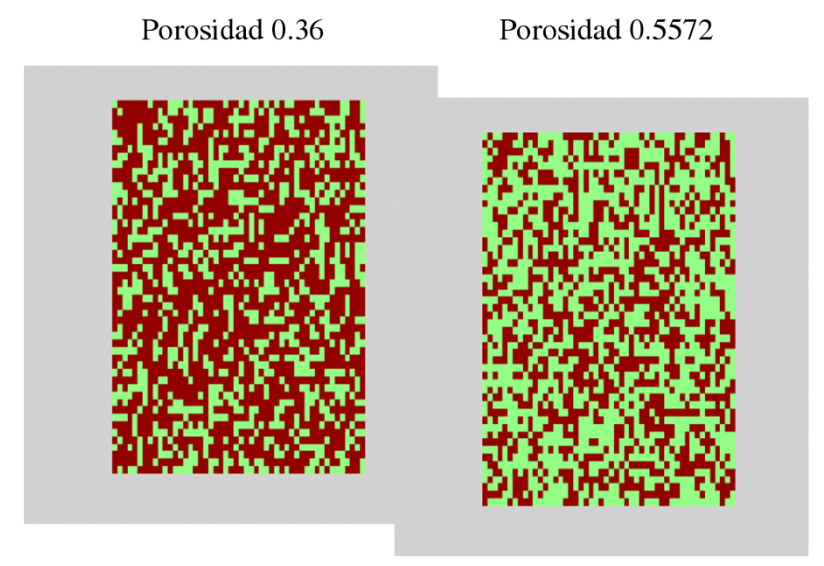

Figura 2: Ejemplos de estructuras con porosidad $b=0.36$ y $b=0.56$.

Se decidió implementar computacionalmente el llamado Modelo A para este tipo de fenómenos, tomando en cuenta los trabajos reportados en la literatura [1], [3], [7], [11], [12].

Ha sido muy útil para la simulación del proceso de adsorción-desorción para fluidos con diferentes concentraciones de elementos, los conceptos desarrollados en [7]. En nuestro caso el modelo no es cerrado dada la interacción con otras zonas del modelo.

En el autómata celular considerado para esta zona se asignan valores probabilísticos de adsorción para cada grano, valores indicativos de ocupación o no de las celdas que representan a los granos y se maneja el valor del porcentaje de concentración del contaminante. La vecindad utilizada es una de Von Neumann. Las reglas que se siguen en el funcionamiento del autómata son las dadas por Merino et al. [7, pág. 1203] "Para la celda $A(i, j), P(i, j)$ es el valor probabilístico de adsorción de la celda $A(i, j)$ y pdada es el porcentaje de concentración del contaminante. Se establece que

i) Si $A(i, j)=1$ y $P(i, j) \geq p d a d a$ y al menos un vecino en 0 entonces $A(i, j)=1$.

ii) Si $A(i, j)=1$ y toda la vecindad ocupada entonces $A(i, j)=1$.

iii) Si $A(i, j)=1$ y $P(i, j) \leq 1-p d a d a$ y al menos un vecino en 0 entonces $A(i, j)=0$.

iv) Si $A(i, j)=0$ y $P(i, j) \leq 1-p d a d a$ entonces $A(i, j)=0$.

v) Si $A(i, j)=0$ y $P(i, j) \geq p d a d a$ entonces $A(i, j)=1$." 
Lo anterior permite generar un autómata celular probabilístico de funcionamiento síncrono.

\subsubsection{Difusión en los poros}

La simulación se realiza con el modelo FHP II [4], el cual utiliza un látice triangular que permite el manejo de 6 partículas con velocidad unitaria y una partícula en reposo en cada nodo. Las partículas tienen masa unitaria y el sistema se actualiza en dos pasos: colisión, propagación.

La ecuación microscópica para las dos operaciones mencionadas es:

$$
N_{a}\left(x+e_{a}, t+1\right)=N_{a}(x, t)+\Omega_{a}
$$

donde $N_{a}$ es el número de partículas en el nodo $x$ en el instante $t$ con momento $e_{a}(a=1, \ldots, 6)$ y $\Omega_{a}$ denota la razón de cambio en el tiempo del número de partículas, debido a las colisiones.

Los parámetros macroscópicos: densidad local promedio $n$ y momento promedio $n u$, se definen:

$$
\begin{aligned}
n & =\sum_{a} f_{a} \\
n u & =\sum_{a} f_{a} e_{a}
\end{aligned}
$$

donde $f_{a}$ es el promedio en la vecindad de $N_{a}(x, t)$.

De acuerdo a lo expuesto por Frisch et al. [4] se manejan las siguientes ecuaciones de Navier-Stokes:

$$
\begin{aligned}
\frac{\partial n}{\partial t}+\nabla \cdot n u & =0 \\
\frac{\partial\left(n u_{\alpha}\right)}{\partial t}+\frac{\partial n g(n) u_{\alpha} u_{\beta}}{\partial x_{\beta}} & =\frac{\partial p}{\partial x_{\beta}}+\frac{\partial\left(\mu^{\partial u_{\alpha} / \partial x_{\beta}}\right)}{\partial x_{\beta}}
\end{aligned}
$$

donde $n$ es la densidad del fluido, $u_{\alpha}$ es la velocidad y $\mu$ es el coeficiente de viscosidad.

El coeficiente del término de convectividad $g(n)$, debe ser unitario para simular un sistema físico continuo. Nótese que $g(n)$ sólo depende de la densidad y de la geometría del modelo de látice de gases cuando la probabilidad de la colisión de transición satisface el balance semidetallado. La presión está dada por:

$$
p^{\cdot}=\frac{3}{7} n\left(1-\frac{5}{6} g(n) u^{2}\right) .
$$

La dependencia de la presión con la velocidad macroscópica causa oscilaciones, las que pueden controlarse [2] si: 
a) se controla la densidad inicial [15] o

b) se utiliza un modelo de látice de gases con velocidades múltiples [14].

En este modelo se utiliza la opción a).

\subsection{Zona del agua}

En esta zona se aplica directamente la ecuación general de difusión:

$$
\partial_{t} \rho(\vec{r}, t)=\operatorname{div}[D(\rho, \vec{r}) \operatorname{grad} \rho(\vec{r}, t)] .
$$

En un principio manejaremos únicamente la difusión del contaminante en el agua.

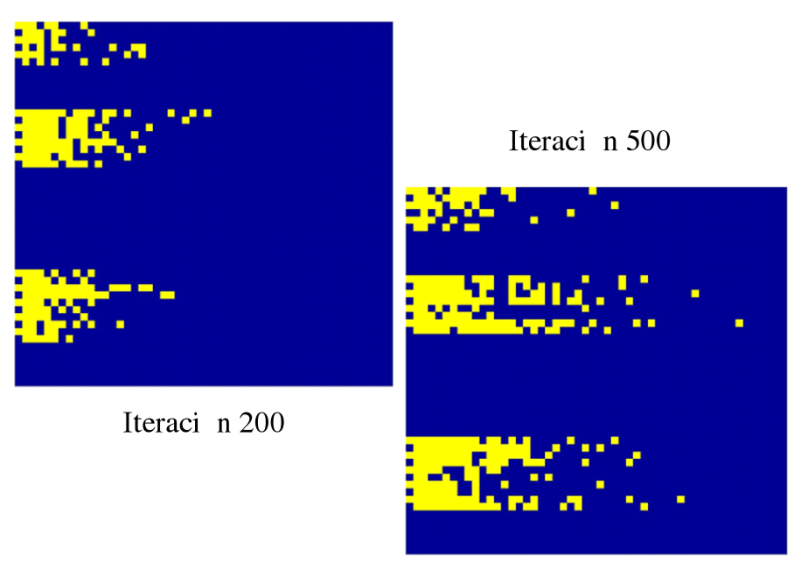

Figura 3: Ejemplos de difusión del contaminante en la zona del agua. En las iteraciones 200 y 500 .

\subsection{Zona de interrelación del suelo y del agua}

Esta es una zona de suma importancia por los diferentes fenómenos físicos que en ella ocurren. Aquí el fluido contaminado entra en contacto con el agua en los poros de la matriz de suelo provocando un movimiento de convección, transporte y disolución del contaminante el que, finalmente, ingresará en el medio acuoso. Por supuesto que se presentan fenómenos de erosión en la orilla del suelo y por lo tanto arrastre de sedimentos en el agua. En la parte inicial del modelo no se considerará el fenómeno de erosión. 
En la parte del suelo además de aplicar las condiciones ya discutidas, se permite la adsorción de agua en el suelo produciendo movimientos de fluido en el sentido de agua a suelo y de suelo hacia agua.

Para el modelado en la parte del agua utilizamos los siguientes operadores:

a) $P$ operador de propagación. El operador $P$ es el operador de propagación de los modelos de látice de gases [4]: cada partícula se mueve de un nodo a otro en la dirección de su velocidad. En esta fase las partículas conservan su velocidad.

b) $R$ operador de rotación. El operador $R$ es un operador estocástico que gira de manera independiente cada nodo $\pi / 2$ en el sentido de las manecillas del reloj o en contra de las manecillas con una probabilidad $P_{r \circ t}$.

La composición $R \circ P$ es el operador de evolución del sistema en el tiempo.

Nótese que con el operador de evolución $R \circ P$, cada partícula ejecuta una caminata aleatoria a través de desplazamientos y rotaciones sucesivas. Estas caminatas aleatorias no son totalmente independientes, por que al operar la rotación en cada nodo, las partículas giran al mismo tiempo sin importar cual sea su velocidad. No obstante, este es un efecto pequeño y en una primera aproximación podemos asumir la independencia de los "caminantes". En el límite, la densidad de la probabilidad $\rho$ de encontrar al caminante $x i$ en el instante $t$ en el sistema, sigue la ecuación de difusión:

$$
\frac{\partial \rho(\xi, t)}{\partial t}=D \Delta \rho(\xi, t)
$$

donde $D$, coeficiente de difusión, está dado por la expresión clásica en dos dimensiones:

$$
D=\lim _{t \rightarrow \infty} \frac{1}{4} \frac{\sigma^{2}(t)}{t}
$$

con $\sigma^{2}(t)$ desplazamiento al cuadrado de una partícula. La relación entre $\sigma^{2}(t)$ y $P_{r \circ t}$ es:

$$
\sigma^{2}(t) \approx_{t \rightarrow \infty} t+2 t \frac{1-P_{r \circ t}}{P_{r \circ t}}
$$

\section{Funcionamiento general del autómata celular}

El Autómata Celular funciona en una matriz de orden $n \times\left(n+n_{1}+n_{2}\right)$; $n_{1}$ es el número de columnas asignadas a la zona de interrelación del suelo y agua; $n_{2}$ es el número de columnas asignadas a la zona de agua. Una 
vez generado el reticulado se sobrepone en las primeras $n$ columnas, la estructura porosa. La actualización del Autómata Celular se realiza de izquierda a derecha con la introducción del flujo del contaminante de arriba hacia abajo, en cada instante discreto del tiempo, excepto en la zona de interrelación del suelo-agua.

Dentro del Autómata Celular las partículas contaminantes que, por el algoritmo al que estén sujetas se muevan hacia las columnas $\left(n+n_{1}+n_{2}\right)$ ó "antes de la 1" y hacia el renglón $n+1$ salen del modelo; el interés se centra en el funcionamiento del modelo dentro de la matriz definida.

El modelo computacional para el funcionamiento general del Autómata Celular se diseñó con base a los módulos correspondientes a cada zona y subzona descritas anteriormente, lo que permite una actualización o modificación de sus características con bastante facilidad. Por ejemplo, la introducción de una zona permeable en la parte inferior del modelo, correspondiente al renglón de la matriz del suelo.

\section{Conclusiones}

El modelo permite:

- Simular el comportamiento del contaminante interactuando con los diferentes elementos del sistema suelo-agua.

- Observar el flujo del contaminante en el sistema suelo-agua.

- Cambiar el tipo de porosidad para diferentes simulaciones.

- Tener la posibilidad de cambiar la concentración del contaminante para diferentes simulaciones.

- La entrada del fluido contaminante con diferentes concentraciones dentro de una misma simulación.

Además,

- Se asume que los extremos izquierdo, derecho e inferior del Autómata Celular marcan el final de la zona de interés para la simulación y las partículas que llegan a dichos extremos salen del modelo.

- Se puede introducir una capa permeable en la parte inferior del autómata celular. Lo anterior introduce una frontera cerrada en el Autómata Celular.

- Se puede observar la evolución del sistema suelo-agua, al dejar funcionar el autómata celular suspendiendo la introducción del fluido contaminante. 
- Este tipo de modelación permite una simulación más rápida de los fenómenos de flujo de contaminantes entre diferentes medios.

\section{Referencias}

[1] Chopard, B.; Droz, M. (1998) Cellular Automata Modeling of Physical Systems. Cambridge University Press, Cambridge MA.

[2] Chen, S.; Diemer, K.; Doolen, G.D.; Eggert, K.; Fu, C.; Gutman, S.; Travis, B.J. (1991) "Lattice gas automata for flow through porous media", Physica D: Nonlinear Phenomena 47(1-2): 72-84.

[3] Bagnoli, F.; Chopard, B.; Droz, M.; Frachebourg, L. 1992) "Critical behaviour of a diffusive model with one adsorbing state", Journal of Physics A: Mathematical and General 25(5): 1085-1091.

[4] Frisch, U.; d'Humières, D.; Hasslacher, B.; Lallemand, P.; Pomeau, Y.; Rivet, J.P. (1990) "Lattice gas hydrodynamics in two and three dimensions", in: G.D. Doolen (Ed.) Lattice Gas Methods for Partial Differential Equations. Addison-Wesley Publishing Company, Redwood City CA: 77-135.

[5] Pape, H.; Clauser, C.; Iffland, J. (1999). "Permeability prediction based on fractal pore-space geometry", Geophysics 64(5): 1447-1460.

[6] Liu, G.; Zheng, C.; Gorelick, S.M. (2004), "Limits of applicability of the advection-dispersion model in aquifers containing connected highconductivity channels", Water Resources Research 40: 1-19.

[7] Merino, G.; Martín, M.; Sánchez, M.; Garbi, C.; Alonso, R. (2002) "Utilización de un Autómata Celular en el modelado de un proceso de descontaminación de aguas", in: E. Oñate, F. Zárate, G. Ayala, S. Botello \& M.A. Morelos (Eds.) Métodos Numéricos en Ingeniería y Ciencias Aplicadas, Vol. 2, CIMNE, Barcelona. Disponible en http://www . cimne. upc. es/congress/gto2002/II\%20Foro/PDF/ V\%20II/Procesos\%20Industriales/Merino1201.pdf

[8] Merino, G.; Bustillos, O. (1998) "Simulation of pollutant difusion between two media by means of cellular automata", VII International Congress of Ecology, Florence, Italy.

[9] Merino, G.; Bustillos, O. (1996) "Autómata celular con diferentes reglas locales", XXIX Congreso de la Sociedad Matemática Mexicana. 
[10] Rieu, M.; Sposito, G. (1991) "Fractal fragmentation, soil porosity and soil water properties: I. Theory", Soil Sci. Soc. Am. J. 55: 1231-1238.

[11] Benney, P.; Droz, M.; Frachebourg, L. (1990) "On the critical behaviour of cellular automata models of non-equilibrium phase transitions", Phys. A: Math. Gen. 23: 3353-3359.

[12] Gaylor, R.J.; Nishidate, K. (1996) Modeling Nature: Cellular Automata Simulations with Mathematica. Springer-Verlag, New York.

[13] Dickman, R.; Burschka, M.A. (1988) "Nonequilibrium critical poisoning in a single-species model", Physics Letters A 127(3): 132-137.

[14] Chen, S.; Chen, H.; Doolen, G.D. (1989) "How the lattice gas model for the Navier-Stokes equation improves when a speed is added", Complex Systems 3: 243-251.

[15] Chen, S.; She, Z.; Harrison, L.C.; Doolen, G.D. (1989) "Optimal initial condition for lattice gas hydrodynamics", Phys. Rev. A 39: 27252727 .

[16] Di Gregorio, S.; Serra, R.; Villani, M. (1997) "A cellular automata model of soil bioremediation", Complex Systems 11: 31-54.

[17] Toffoli, T.; Margolous, N. (1991) Cellular Automata Machines. A New Environmental for Modeling, Fifth printing. MIT Press, Cambridge MA.

[18] Pachepsky, Ya.A.; Korsunskaia, L.P.; Hajnos, M. (1996) "Fractal parameters of soil pore surface area under a developing crop", Fractals 4(1): 97-104.

[19] Efendiev, Y.; Durlofsky, L.J. (2003) "A generalizad convectiondiffusion model for subgrid transport in porous media", SIAM Multiscale Modeling and Simulation 1(3): 504-526. 